\title{
Analysis of differentially expressed genes in white blood cells isolated from patients with major burn injuries
}

\author{
GONGJIE TANG ${ }^{1}$, TAO ZHANG ${ }^{1}$, XINBO WANG $^{1}$, ZENGMEI SONG $^{1}$, \\ FUCUN LIU ${ }^{1}$, QIAN ZHANG ${ }^{1}$ and RAN HUO ${ }^{2}$ \\ ${ }^{1}$ Department of Burn and Plastic Surgery, Linyi Central Hospital, Linyi, Shandong 276400; ${ }^{2}$ Department of \\ Burn and Plastic Surgery, Shandong Provincial Hospital, Jinan, Shandong 250011, P.R. China
}

Received May 25, 2017; Accepted July 24, 2017

DOI: $10.3892 /$ etm.2017.4899

\begin{abstract}
The aim of the present study was to identify differentially expressed genes (DEGs) and their related functions and pathways of major burn injuries, and to prevent the occurrence of complications. The expression profiling of E-GEOD-37069 was downloaded from ArrayExpress Archive. The DEGs of major burn injuries were identified. Gene Ontology (GO) and Kyoto Encyclopedia of Gene and Genomes (KEGG) functional enrichment analysis were then performed for the DEGs. Based on the KEGG database, a pathway relationship network was constructed, and DEGs in significant GO terms and pathways were investigated. Gene signal network and gene co-expression network of these inserted DEGs were constructed. A total of 3,328 DEGs of major burn injuries were identified, including 1,337 up- and 1,991 downregulated DEGs. These DEGs were mainly enriched into various $\mathrm{GO}$ terms, including transcription, DNA-dependent, signal transduction and blood coagulation. Moreover, they were also enriched into different pathways, such as hematopoietic cell lineage, metabolic pathway and chemokine signaling pathway. The pathway relationship network was constructed with 72 nodes. The MAPK signaling pathway was the hub node. Based on the same gene symbol, 702 DEGs were obtained, identified in both GO terms and pathways. Finally, the gene signaling network and gene co-expression network were constructed with 391 and 128 nodes, respectively. These identified DEGs, including GNB2, LILRA2, ARRB2 and ARHGEF2, may be potential key genes involved in the treatment of major burn injuries and prevention of complications.
\end{abstract}

\section{Introduction}

Major burn injuries constitute a systemic disease that may induce post-traumatic stress disorder and pain and further

Correspondence to: Dr Ran Huo, Department of Burn and Plastic Surgery, Shandong Provincial Hospital, 324 Jingwu Road, Shizhong, Jinan, Shandong 250011, P.R. China

E-mail: huoran1a2b3c@163.com

Key words: gene expression analysis, major burn injures, differently expressed genes effect functioning and disability (1). In addition, major burn injuries cause capillary fluid leakage and tissue swelling, and require large amounts of intravenous infusion (2). The most common complications of major burn injuries involve shock, insufficient pulmonary functions, pneumonia and acute renal failure (3). However, these complications, especially infection, constitute a significant challenge in post-burn care. Thus, investigation of post-burn genetic variability is imperative to determine treatment for burn injuries and possible complications (4). Genetic variability following major burn injury has been previously identified, including potential genes involved in the immune-inflammatory response (5). In addition, the expression level of IL- 8 in serum was confirmed to be an effective biomarker for detecting sepsis, infections and mortality after major burn (6). Holmes et al (7) determined that cholinergic mediator and expression of HMGB1 and caspase-3 were closely related to post-burn infection and graft failure. In skin burn injury, the differential expression of various genes, including interleukin (IL)-10, transforming grow th factor (TGF)- $\beta$, MCP-1 and MMP- 2 was increased (8). Even in human skeletal muscles, genetic variability of the TWEAK family was increased following major burn injury (9). Nevertheless, the obtained genes were few and their molecular mechanisms were not clarified. Thus, postburn-related genes and their systematic relations need to be investigated.

The aim of the present study was to identify differentially expressed genes (DEGs) and their related functions and pathways of major burn injuries, and to lay a theoretical basis for the treatment of such injuries and prevention of possible complications. For this purpose, DEGs in white blood cell samples obtained from patients with major burn injuries compared with normal controls were screened, and functional and pathway enrichment analyses were processed. Finally, various networks including a pathway relationship network, gene signaling network and gene co-expression network were constructed.

\section{Materials and methods}

Samples. The expression profiling of E-GEOD-37069 deposited by Herndon et al and including 590 white blood cell samples, was downloaded from ArrayExpress Archive 
(http://www.ebi.ac.uk/arrayexpress/), which was based on the platform of [HG-U133_Plus_2] Affymetrix Human Genome U133 Plus 2.0 Array (Affymetrix, Santa Clara, CA, USA) (6). The samples included 553 patients with major burn injuries and 37 controls. The raw data profiling of 590 samples were further analyzed for DEGs screening of major burn injuries.

Data preprocessing and DEGs screening. For raw data, the robust multichip average (RMA) algorithm was used to calculate the expression value of probe sets in three steps: Background correction, normalization and summary (10). The annotation file in csv format, provided by Affymetrix (http:// www.affymetrix.com/support/technical/annotationfilesmain.affx), was used for the biological information annotation. Subsequently, quality control was performed by normalized unscaled standard errors (NUSE) controlling.

The DEGs in white blood cell samples with major burn injuries compared with normal controls were screened using a limma package. $\mathrm{P}<0.05$ and $\log \mathrm{FCl}>2$ were regarded as cut-off criteria of DEGs.

Functional enrichment analysis for DEGs. Based on the Gene Ontology (GO) database, the screened DEGs were annotated and classified into various functional categories, including molecular function (MF), cellular component (CC) and biological process (BP) (11). Fisher's exact test was used to calculate the P-value of each GO term. The Beniamini-Hochberg ( $\mathrm{BH})$ method was performed to adjust the P-values into false discovery rate (FDR). FDR $<0.05$ was the cut-off criterion for significant GO terms.

Pathway enrichment analysis and pathway relationship network construction. The pathway data were obtained from the Kyoto Encyclopedia of Gene and Genomes (KEGG) (12). Based on this database, different pathways were gathered by DEGs, and their P-values were calculated by Fisher's exact test with a threshold of $\mathrm{P}<0.05$.

In addition, the significant pathways were compared to the KEGG database, and the pathway relationship network was constructed. The network showed the pathway relationship of signal transduction. In addition, the up- and downstream signal pathways were obtained in this network.

Intersection of DEGs in GO terms and pathways. Based on the same gene symbols, DEGs in significant GO terms and pathways were interested and common DEGs were obtained as more important DEGs.

Construction of gene signaling network and gene co-expression network. The KEGG database provides information regarding associations among genes and their productions. In this study, the gene signaling network was constructed based on this database. Furthermore, up- and downregulated proteins were showed in this network.

Gene co-expression network was a regulatory network for genes interactions. This network was constructed according to the correlation coefficient between common DEGs. Additionally, a correlation coefficient of $>1$ was the criterion. In the constructed gene co-expression network, DEGs with higher degrees were regarded as hub nodes.

\section{Results}

DEGs screening. Based on the threshold of DEGs, 3,328 DEGs in patients with major burn injuries compared with controls were identified, including 1,337 upregulated DEGs and 1,991 downregulated DEGs.

Functional enrichment analysis for DEGs. For functional enrichment analysis, the identified DEGs were mainly enriched into various GO terms, including transcription, DNA-dependent $(F D R=1.81 \mathrm{E}-34)$, signal transduction (FDR=6.41E-32), blood coagulation $(\mathrm{FDR}=6.41 \mathrm{E}-32)$, regulation of transcription, and DNA-dependent (FDR=4.97E-30). The enrichment scores of these GO terms were 2.50, 3.02, 4.26 and 0.69 , respectively.

Pathway enrichment analysis and pathway relationship network construction. In addition, the screened DEGs were enriched into different pathways, such as hematopoietic cell lineage $(\mathrm{FDR}=7.40 \mathrm{E}-17)$, metabolic pathway $(\mathrm{FDR}=7.40 \mathrm{E}-17)$ and chemokine signaling pathway (FDR=9.37E-17).

Furthermore, the pathway relationship network was constructed with 72 nodes. Of these nodes, MAPK signaling pathway, apoptosis and pathways in cancer were the top three hub nodes with degrees of 41, 32 and 25, respectively (Fig. 1). Of note, there were 2 upregulated pathways and 4 downregulated pathways. Upregulated pathways contained starch and sucrose metabolism (degree $=3$ ) and fructose and mannose metabolism (degree $=1$ ), while the downregulated pathways included graftversus-host disease (degree $=7$ ), autoimmune thyroid disease (degree=6), allograft rejection (degree=6) and type I diabetes mellitus (degree=2). Pathways in cancer constituted significant upstream pathways (outdegree $=25$, indegree $=0$ ).

Intersection of DEGs in GO terms and pathways. Based on the same gene symbol, 702 DEGs were obtained, as identified in both GO terms and pathways. The obtained common DEGs included ribosomal protein L31 (RPL31), ATP synthase, $\mathrm{H}+$ transporting, mitochondrial $\mathrm{F} 1$ complex, $\gamma$ polypeptide 1 (ATP5C1), phosphorylated adaptor for RNA export (PHAX) and solute carrier family 4 member 1 (SLC4A1).

Construction of gene signaling network and gene co-expression network. As shown in Fig. 2, the gene signaling network with 391 nodes was constructed. The top three hub nodes in this network were phosphatidylinositol-4,5-bisphosphate 3-kinase catalytic subunit $\gamma(\mathrm{PIK} 3 \mathrm{CG}$, degree $=40)$, protein kinase CAMP-activated catalytic subunit $\beta$ (PRKACB, degree=17) and AKT serine/threonine kinase 3 (AKT3, degree=12). From this network, various DEGs and PIK3CG were activated, such as $\mathrm{Cbl}$ proto-oncogene (CBL), G protein subunit $\beta 2$ (GNB2) and CD28 molecule (CD28). Moreover, PRKACB interacted with phosphorylated $G$ protein subunit $\beta$ (GNB) family genes.

The constructed gene co-expression network is shown in Fig. 3. The gene co-expression network had 128 nodes. The top 3 nodes were GNB2 (degree=35), leukocyte immunoglobulin like receptor A2 (LILRA2, degree $=31)$ and arrestin $\beta 2($ ARRB2, degree=30). In addition, GNB2, LILRA2 and ARRB2 were positively correlated with the gene co-expression network. 


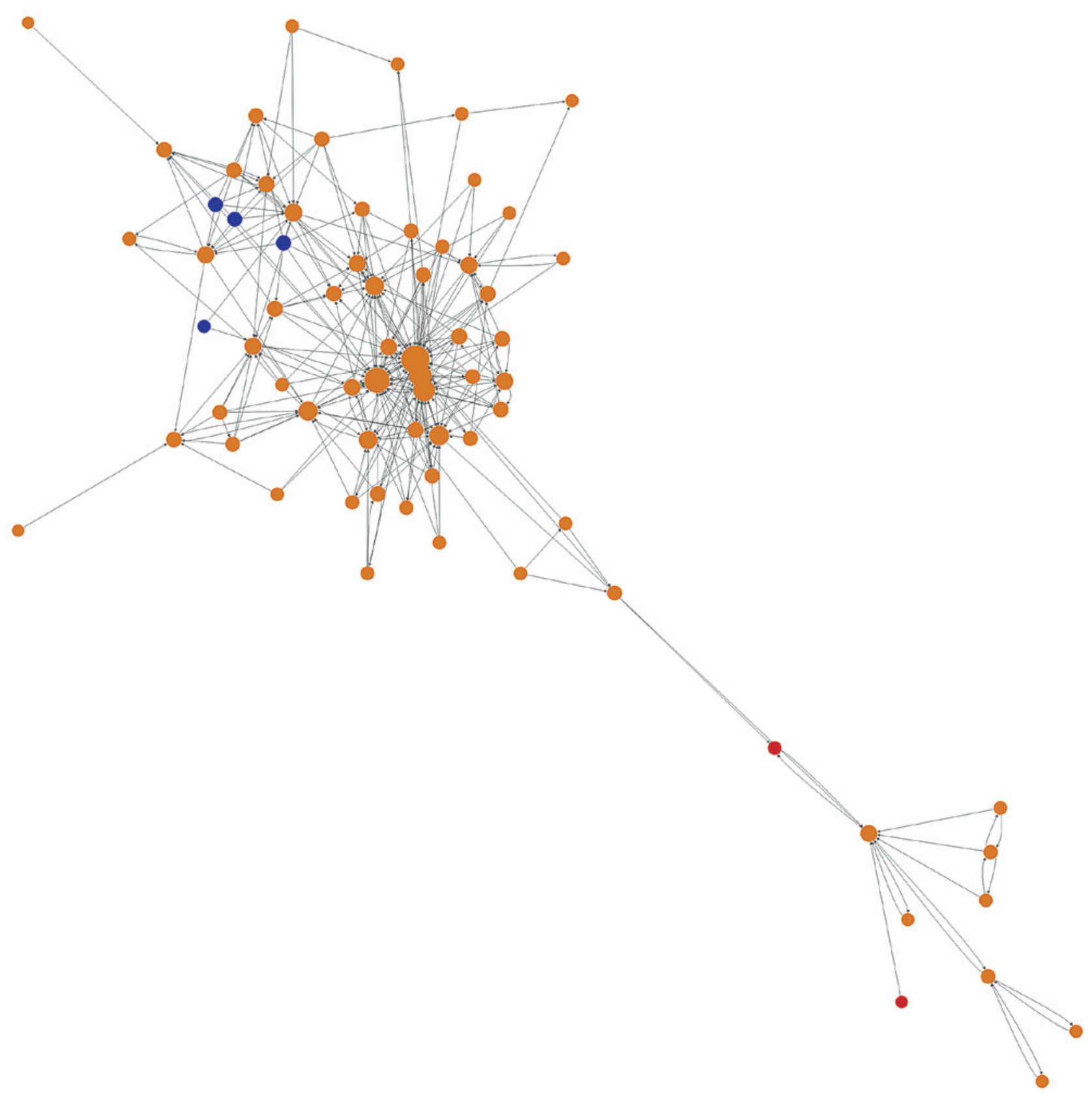

Figure 1. The pathway relationship network of major burn injuries. The nodes are significant pathways, while the edges are their relationships. The red nodes involved upregulated DEGs, while the blue nodes involved downregulated DEGs. The yellow nodes involved both up- and downregulated DEGs. DEGs, differentially expressed genes.

\section{Discussion}

The changes in the expression levels induced by major burn injuries may cause serious consequences, such as various complications. In this study, several important DEGs were identified in major burn injury patients, including GNB2, LILRA2, ARRB2 and ARHGEF2.

GNB2, encoded heterotrimeric guanine nucleotide-binding proteins ( $G$ protein), integrates signals between receptors and effector proteins (13). It is known that the protein coded by GNB2 is present in extracellular, lysosome and vacuole. In addition, $\mathrm{G}$ protein acted as a molecular switch, is involved in the transmission of signaling pathways, and may be coupled with GTP or GDP (14). After burn injuries, immune responses are always controlled by formyl peptide receptors, a promiscuous subfamily of $\mathrm{G}$ protein-coupled receptors (15). In addition, Gao et al (16) identified the effects of tetramethylpyrazine on burn-injury models and found that this drug alleviated nociceptive transmission mediated by $\mathrm{P} 2 \mathrm{X} 3$ receptor. In this study, GNB2 was enriched into the GTP catabolic process, $\mathrm{G}$ protein-coupled receptor signaling pathway and chemokine signaling pathway. In rat module with sepsis, $G$ protein was confirmed as a major component of GTP-binding protein involved in signal transduction pathway (17). Thus, GNB2 was differentially expressed in major burn injury patients and may affect the treatment and possible complications occurring from burn injury.

Furthermore, ARRB2 and GNB2 were positively correlated with the gene co-expression network of this study. The protein coded by ARRB2 in a previous study was 


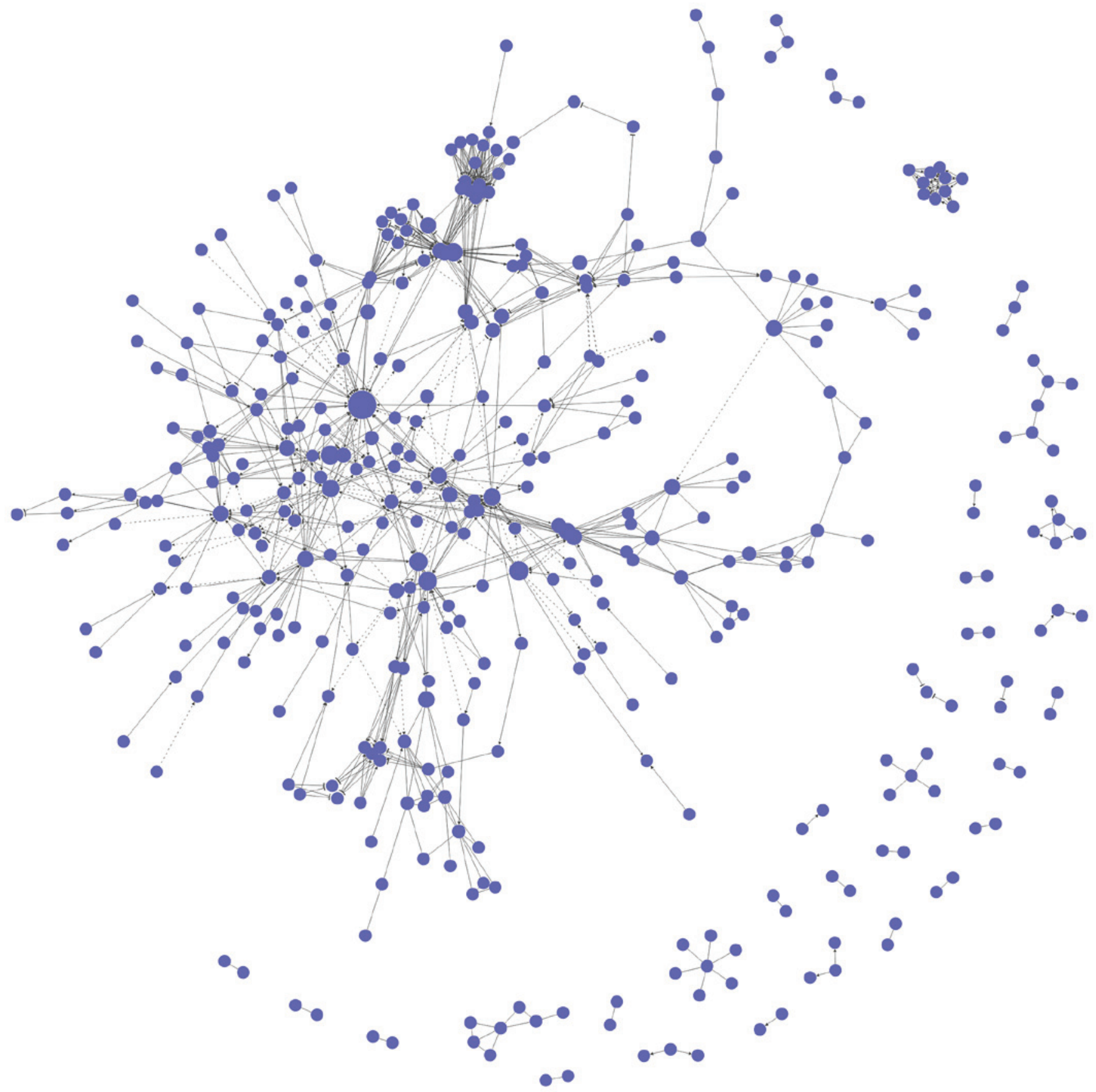

Figure 2. Gene signal network of common DEGs. The nodes and edges are common DEGs and their relationships, respectively. DEGs, differentially expressed genes.

confirmed to participate in agonist-mediated desensitization of $G$ protein-coupled receptors and to induce the specific dampening of cell responses (18). In addition, ARRB2 was more highly expressed in the central nervous system, and also regulated the synaptic receptors (19). Additionally, shock, a common complication of major burn injuries, was found to be closely related to the regulation of receptors, sensitivity to neurotransmitters and non-synaptic transmission (20). Consistent with the results of the present study, ARRB2 was involved in signal transduction, platelet activation, as well as the chemokine and MAPK signaling pathways. Cytokine and chemokine of inflammatory response was the common response behavior for burn injury. Through a drug trial,
Kim et al (21) found that fimasartan reduced renal oxidative stress by inhibiting MAPKs and induced antioxidant pathways. Therefore, differentially expressed ARRB2 induced by major burn injuries may further cause renal failure by its involvement in the MAPK signaling pathway.

Notably, LILRA2 was positively correlated with the DEGs examined in this study. The gene encoded an activating receptor which suppressed the innate immune response and inhibited antigen presentation (22). Moreover, it was suggested to mediate activation of monocyte and inhibit Fc $\gamma$ RI-dependent phagocytosis (23). In 1998, Witko-Sarsat et al found that advanced oxidation protein products were a mediator between monocyte respiratory burst and oxidative stress, and confirmed 


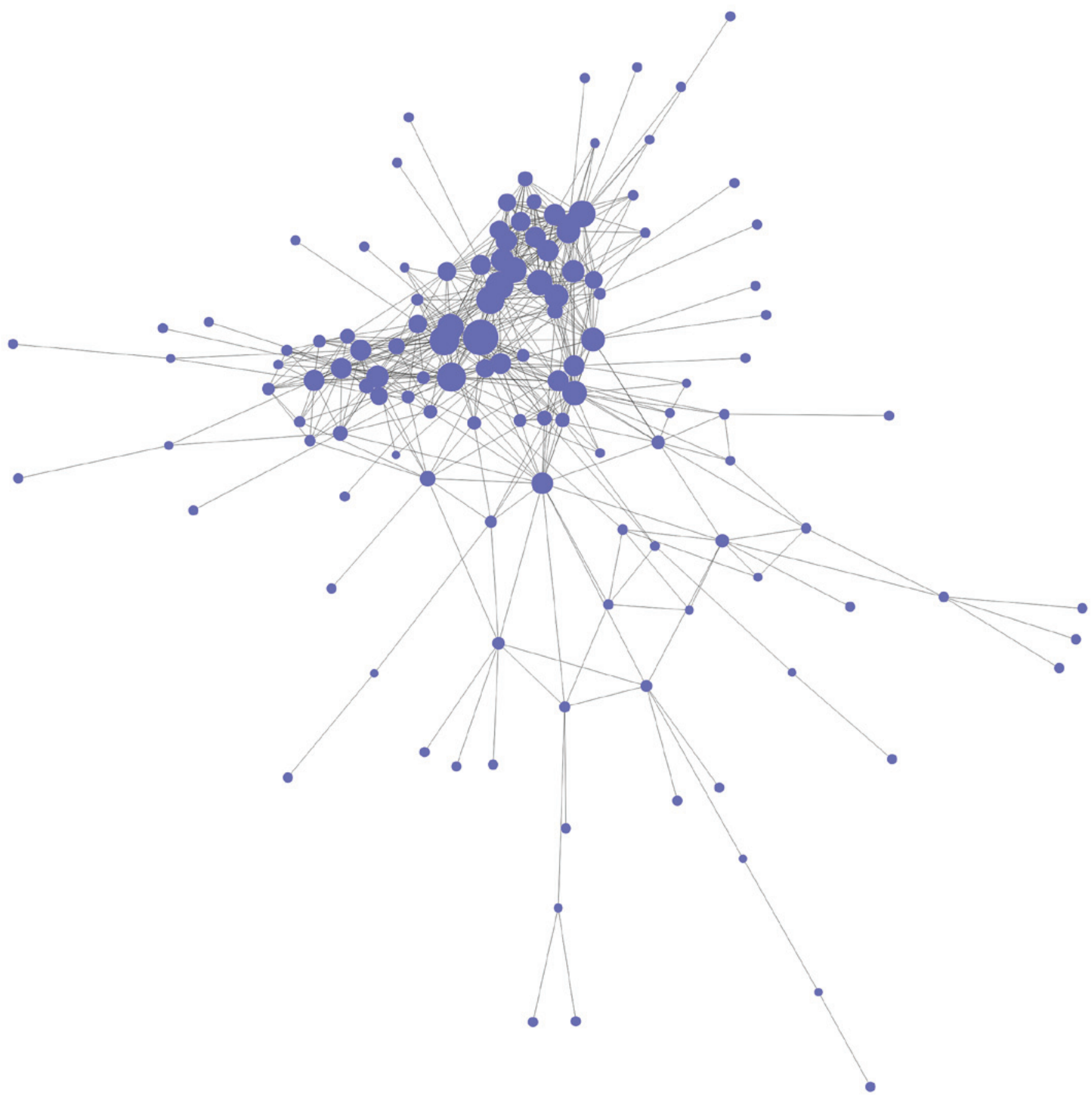

Figure 3. Gene co-expression network of common DEGs. The nodes and edges are common DEGs and their relationships, respectively. DEGs, differentially expressed genes.

that monocytes were the key target and an actor in the immune system related to renal failure (24). In this study, LILRA2 was also enriched in signal transduction, defense response and osteoclast differentiation. Previous findings showed the various defense response was related to burn complications, such as antioxidative defense of lipoproteins and erythrocyte defense $(25,26)$. The findings suggested that LILRA2 is a potential biomarker for complication of major burn injuries.

Another significant DEG, ARHGEF2 was identified with higher degree of correlation in our gene co-expression network. In addition, it was enriched into apoptotic process, small GTPase-mediated signal transduction and pathogenic Escherichia coli infection. ARHGEF2 encoded a Rho GTPases which play a fundamental role in numerous cell processes (27). Koh found that the Rho GTPases played a significant role in the function and morphology of the neurons (28). In addition, the intestinal permeability in major burn patients were always related to infection (29). Mortality in major burn patients was always caused by bacterial infections (30). Thus, the detection of ARHGEF2 in time could decrease the complication occurrence and mortality.

In conclusion, the identified DEGs, including GNB2, LILRA2, ARRB2 and ARHGEF2, are potential key genes for major burn injury treatment and the prevention of possible complications. However, further experiments are required to confirm these results. 


\section{Acknowledgements}

This study did not receive any specific grant from funding agencies in the public, commercial or not-for-profit sectors.

\section{References}

1. Corry NH, Klick B and Fauerbach JA: Posttraumatic stress disorder and pain impact functioning and disability after major burn injury. J Burn Care Res 31: 13-25, 2010.

2. Yeung J, Yeung T and Yeung A: Disc shunt delivery with stepped needle. US 20120065570 A1. Filed September 12, 2011; issued March 15, 2012.

3. Nainar P, Baker HV, Lopez C, Tomkins RG, Herndon D, Finnerty CC, Kudlicki AS, Fongang B, Li X, Arnoldo BD, et al: Differential Wbc gene expression is associated with pneumonia development after burn injury. Shock 41: 57-58, 2014.

4. Rowley-Conwy G: Infection prevention and treatment in patients with major burn injuries. Nurs Stand 25: 51-54, 2010.

5. Schwacha MG, Holland LT, Chaudry IH and Messina JL: Genetic variability in the immune-inflammatory response after major burn injury. Shock 23: 123-128, 2005.

6. Kraft R, Herndon DN, Finnerty CC, Cox RA, Song J and Jeschke MG: Predictive value of IL- 8 for sepsis and severe infections after burn injury: A clinical study. Shock 43: 222-227, 2015.

7. Holmes CJ, Plichta JK, Gamelli RL and Radek KA: Burn injury alters epidermal cholinergic mediators and increases HMGB1 and caspase 3 in autologous donor skin and burn margin. Shock 47: 175-183, 2017.

8. Kubo H, Hayashi T, Ago K, Ago M, Kanekura T and Ogata M: Temporal expression of wound healing-related genes in skin burn injury. Leg Med (Tokyo) 16: 8-13, 2014.

9. Merritt EK, Thalacker-Mercer A, Cross JM, Windham ST, Thomas SJ and Bamman MM: Increased expression of atrogenes and TWEAK family members after severe burn injury in nonburned human skeletal muscle. J Burn Care Res 34 e297-e304, 2013

10. Irizarry RA, Bolstad BM, Collin F, Cope LM, Hobbs B and Speed TP: Summaries of affymetrix GeneChip probe level data. Nucleic Acids Res 31: e15, 2003.

11. Wu H, Zhang Y, Zhang W, Pei X, Zhang C, Jia S and Li W: GO categories of biological process (BP), cellular component (CC) and molecular function (MF) for the late water-stress responding genes (P-24h vs. P-0h) in A. sparsifolia primary roots. Figshare. doi: https://doi.org/10.1371/journal.pone.0120791.g010.

12. Wixon J and Kell D: The Kyoto encyclopedia of genes and genomes - KEGG. Yeast 17: 48-55, 2000.

13. Cárdenas-Navia LI, Cruz P, Lin JC, Rosenberg SA and Samuels Y; NISC Comparative Sequencing Program: Novel somatic mutations in heterotrimeric $\mathrm{G}$ proteins in melanoma. Cancer Biol Ther 10: 33-37, 2010.

14. Ross EM: G protein-coupled receptors: Multi-turnover GDP/GTP exchange catalysis on heterotrimeric $\mathrm{G}$ proteins. Cell Logist 4: e29391-e29391, 2014.

15. Migeotte I, Communi D and Parmentier M: Formyl peptide receptors: A promiscuous subfamily of $\mathrm{G}$ protein-coupled receptors controlling immune responses. Cytokine Growth Factor Rev 17: 501-519, 2006.
16. Gao Y, Xu C, Yu K, Li G, Wan F, Liu S, Lin J, Liu H, Zhang J, Li X, et al: Effect of tetramethylpyrazine on DRG neuron P2X3 receptor involved in transmitting pain after burn. Burns 36: 127-134, 2010.

17. Wu LL, Yang SL, Yang RC, Hsu HK, Hsu C, Dong LW and Liu MS: G protein and adenylate cyclase complex-mediated signal transduction in the rat heart during sepsis. Shock 19: 533-537, 2003.

18. Oda Y, Kanahara N, Kimura H, Watanabe H, Hashimoto K and Iyo M: Genetic association between $G$ protein-coupled receptor kinase $6 / \beta$-arrestin 2 and dopamine supersensitivity psychosis in schizophrenia. Neuropsychiatr Dis Treat 11: 1845-1851, 2015.

19. Catalog Number: PA: Polyclonal Anti-ARRB2 Antibody.

20. Bach-y-Rita P and Illis LS: Spinal shock: Possible role of receptor plasticity and non synaptic transmission. Paraplegia 31: 82-87, 1993.

21. Kim S, Kim SJ, Yoon HE, Chung S, Choi BS, Park CW and Shin SJ: Fimasartan, a novel angiotensin-receptor blocker, protects against renal inflammation and fibrosis in mice with unilateral ureteral obstruction: The possible role of Nrf2. Int J Med Sci 12: 891-904, 2015.

22. Lee DJ, Sieling PA, Ochoa MT, Krutzik SR, Guo B, Hernandez M, Rea TH, Cheng G, Colonna M and Modlin RL: LILRA2 activation inhibits dendritic cell differentiation and antigen presentation to T cells. J Immunol 179: 8128-8136, 2007.

23. Lu HK, Mitchell A, Endoh Y, Hampartzoumian T, Huynh O, Borges L, Geczy C, Bryant K and Tedla N: Correction: LILRA2 selectively modulates LPS-mediated cytokine production and inhibits phagocytosis by monocytes. PLoS One 7: 65, 2012

24. Witko-Sarsat V, Friedlander M, Nguyen Khoa T, CapeillèreBlandin C, Nguyen AT, Canteloup S, Dayer JM, Jungers P, Drüeke $\mathrm{T}$ and Descamps-Latscha $\mathrm{B}$ : Advanced oxidation protein products as novel mediators of inflammation and monocyte activation in chronic renal failure. J Immunol 161: 2524-2532, 1998.

25. Schulz T, Schiffl H, Scheithe R, Hrboticky N and Lorenz R: Preserved antioxidative defense of lipoproteins in renal failure and during hemodialysis. Am J Kidney Dis 25: 564-571, 1995.

26. Durak I, Akyol O, Başeşme E, Canbolat O and Kavutçu M: Reduced erythrocyte defense mechanisms against free radical toxicity in patients with chronic renal failure. Nephron 66: 76-80, 1994.

27. Petrie R and Lamarche-Vane N: Rho GTPases. In: Encyclopedia of Neuroscience. Binder MD, Hirokawa N and Windhorst U (eds). Springer, Berlin, pp3541-3544, 2009.

28. Koh CG: Rho GTPases and their regulators in neuronal functions and development. Neurosignals 15:228-237, 2007.

29. Ziegler TR, Smith RJ, O'Dwyer ST, Demling RH and Wilmore DW: Increased intestinal permeability associated with infection in burn patients. Arch Surg 123: 1313-1319, 1988.

30. Maile R, Jones S, Pan Y, Zhou H, Jaspers I, Peden DB, Cairns BA and Noah TL: Association between early airway damage-associated molecular patterns and subsequent bacterial infection in patients with inhalational and burn injury. Am J Physiol Lung Cell Mol Physiol 308: L855-L860, 2015. 